\title{
Computer-Assisted Anatomical Placement of a Double-Bundle ACL through 3D-Fitting of a Statistically Generated Femoral Template Into Individual Knee Geometry
}

\author{
J.W.H. Luites ${ }^{1 \mathrm{a}}$, A.B. Wymenga ${ }^{1 \mathrm{~b}}$, M. Sati $^{2}$, Y. Bourquin ${ }^{2}$, L. Blankevoort ${ }^{3}$, R. van \\ der Venne ${ }^{4 a}$, J.G.M. Kooloos ${ }^{4 b}$ and H.-U. Stäubli ${ }^{5}$ \\ ${ }^{1 \mathrm{a}}$ SMK-Research, ${ }^{1 \mathrm{~b}}$ Dept. of Orthopaedic Surgery, St. Maartenskliniek, \\ P.O. 9011, 6500 GM Nijmegen, The Netherlands \\ a.wymenga@maartenskliniek.nl \\ ${ }^{2}$ Maurice E. Muller Institute for Biomechanics, University of Bern, Switzerland \\ ${ }^{3}$ Orthopaedic Research Center Amsterdam, Academic Medical Center, \\ University of Amsterdam, Amsterdam, The Netherlands \\ ${ }^{4}$ Orhopaedic Research Laboratory, ${ }^{4 \mathrm{~b}}$ Dept. of Anatomy and Embryology, \\ University of Nijmegen, Nijmegen, The Netherlands \\ ${ }^{5}$ Dept. of Orthopaedic Surgery, Surgical Clinic Tiefenauspital, Bern, Switzerland
}

\begin{abstract}
Femoral graft placement is an important factor in the success of ACL-reconstruction. Besides improving the accuracy of femoral tunnel placement, Computer Assisted Surgery (CAS) can be used to determine the anatomic location. This requires a $3 \mathrm{D}$ femoral template with the position of the anatomical ACL-center, based on endoscopical measurable landmarks. This study describes the development and application of this method. The template is generated through statistical shape analysis of the ACL-insertion, with respect to the anteromedial- (AMB) and posterolateral bundle (PLB). The data is mapped onto a cylinder and related to the intercondylar notch surface and the cartilage border on the lateral notch wall $(n=33)$. The template was programmed in a computer-assisted system for ACL-replacement and validated. The program allows real-time tracking of the femur and interactive digitization under endoscopic control. In a wizard-like fashion the surgeon is guided through steps of acquiring the landmarks for the template alignment. The AMBand PLB-center are accurate positioned within 1-3 $\mathrm{mm}$ of the anatomic insertion-centers in individual knees.
\end{abstract}

\section{Introduction}

The anterior cruciate ligament (ACL) consists of two functional bundles, each with its anatomic insertion area [1]. The anteromedial bundle (AMB) is most tight in flexion and the posterolateral bundle (PLB) is tighter near extension [2]. An ACLreconstruction is aimed at obtaining normal knee laxity and kinematics. However, current ACL-reconstruction aims at the repair of only the AMB through an isometric single-bundle technique. A reconstruction with two bundles approximates normal ACL function near extension and in full flexion by repairing both AMB and PLB.

S.L. Delp, A.M. DiGioia, and B. Jaramaz (Eds.): MICCAI 2000, LNCS 1935, pp. 1096-1103, 2000.

(C) Springer-Verlag Berlin Heidelberg 2000 
Restoration of knee kinematics also depends on accurate graft placement. Femoral attachments affect graft length changes most and incorrect positioned femoral tunnels lead to malfunctioning and graft failure [3]. Using a double-bundle technique requires anatomical placement. Different methods for anatomic femoral tunnel localization have been developed [4-6]. Besides the lack of the distinction between the AMB and PLB, most techniques are 2D approaches. Computer Assisted Surgery (CAS) can improve femoral tunnel placement accuracy [7-11], but can also be used to determine the 3D anatomical ACL-insertion center. This requires the development of an averaged template with the $3 \mathrm{D}$ positions of the anatomical AMB and PLB center.

\subsection{Goal}

Development, incorporation in a CAS system and validation of a 3D femoral template with the anatomical ACL-, AMB- and PLB centers, to improve accurate and anatomical femoral placement of a double bundle ACL-graft into the individual knee.

This work describes the computer-assisted placement of the position of the anatomical ACL-center with respect to the AMB and PLB, through 3D fitting of a statistically generated template, based on 2 endoscopical measurable landmarks into individual femoral knee geometry.

\section{Methods}

\subsection{Development}

The template is generated through statistical shape analysis of the ACL-insertion, with respect to the AMB and PLB. 33 Cadaver knees were dissected till the ligaments. During knee movement the fibers of the ACL were divided. The part that was slack in flexion, the PLB, was separated with a sharp scalpel from the fibers that were tight in that position, the AMB. The PLB tensioned near extension. After marking the outlines of both insertion sites with leaded wire, the bundles were dissected.

With an electromagnetic 3Space Fastrak system (Polhemus Navigation Sciences, USA) the 3D positions of points distributed over the insertion outlines (ACL, AMB and PLB) were measured. To relate the insertion sites to the knee geometry, two major landmarks were also 3D measured; Points on the surface of the intercondylar notch and on the cartilage bone-transition at the medial wall of the lateral femoral condyle. (Fig. 1.)

To develop the 3D template, the notch surface geometry of each femur was approximated by a cylinder. The alignment and radius of the cylinder were determined by a least-squares fit on the notch surface data. The cartilage border determined rotation about and location along the cylindrical axis. (Fig. 2.) 

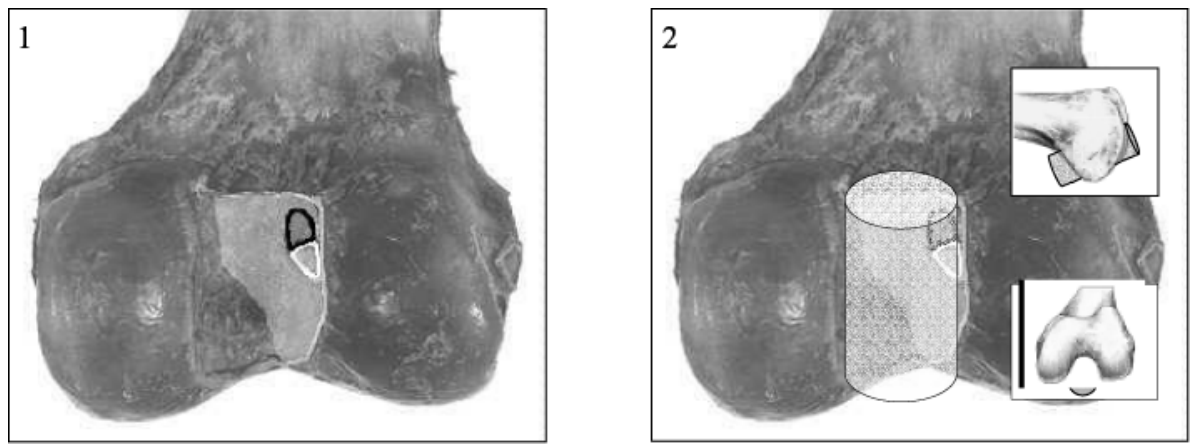

Fig. 1. Left: Dorsal view of the femoral intercondylar notch with the areas 3D digitized by Fastrak: the lateral notch surface, the cartilage border of the lateral condyle and the outlines of the AMB (black) and PLB (white) insertion sites

Fig. 2. Right: The cylindrical fit into the notch surface in dorsal view, lateral (upper inset) and notch view (lower inset)
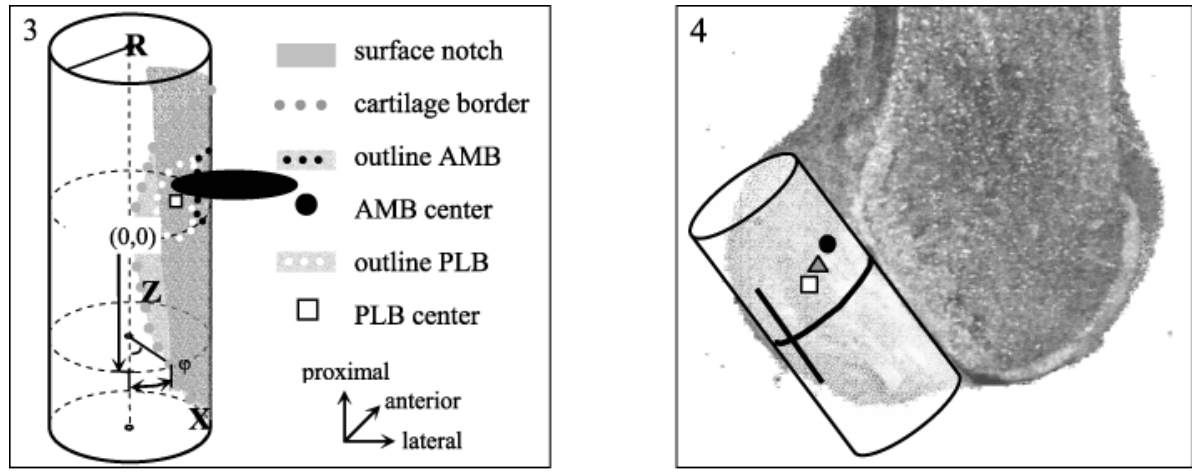

Fig. 3. Left: Ventrolateral view of the fitted cylinder with the projected data of the cartilage border and the AMB- and PLB-insertion outlines. The ACL, AMB and PLB centers were calculated. A parabolic curve was fitted through the cartilage border. The top of the parabola was defined as the origin at the cylindrical surface. From each projected point, the distance in axial (Z) and in horizontal direction at the cylindrical surface (X) (as a result of angle $\varphi$ times mean cylinderradius $\mathrm{R}$ ) was calculated in $\mathrm{mm}$

Fig. 4. Right: Lateral view of a femur with dissected medial condyle. The alignment of the 3D cylinder template with projected on the surface: the origin of the coordinate system (apex of the cartilage border) and the mean position of the centers of the ACL (gray triangle), AMB (black round) and PLB (white square) 
The cartilage-bone transition data and the calculated ACL, AMB and PLB insertion centers were superimposed on the cylinder. A $2^{\circ}$ polynomial was fit through all cartilage-bone transition data by a least squares fit, thus resulting in a function with which the cartilage border was described as a parabola on the cylinder. The apex of the cartilage border curve, i.e. the top of the parabola was defined as the origin on the cylinder surface. The distance in axial direction $(Z)$ between each projected point and the origin was calculated, as was the angle $(\varphi)$. The distance at the cylindrical surface (X) was calculated as a result of the multiplication of the angle with the mean cylinder radius (R). (Fig. 3.)

After scaling all data to the mean cylinder radius of $10.5 \mathrm{~mm}$, the positions of the 33 ACL, AMB and PLB insertion centers, relative to their origin (the apex of the cartilage border curve), were mapped onto the average cylinder. The mean positions of the centers were averaged, thus resulting in a mean femoral template. (Fig. 4)

\subsection{Incorporation}

The femoral template was incorporated in a developed computer assisted system developed at the M.E. Müller Institute for Biomechanics to perform intra-operative planning for ACL replacement. It allows real-time tracking of the femur and tibia and interactive digitization and labeling of anatomical structures identified under direct visual or endoscopic control [7].

\subsection{Validation}

The CAS system was first tested on the population of 33 knees that was used to define the template by comparing the anatomically marked bundle locations to those found by numerical template fit.

In the femora first the outlines of the insertion sites were digitized. The center of the outline was defined as the mean value of the $3 \mathrm{D}$ positions of all measured points. Then the developed program was applied to the femora. The obtained data of the $3 \mathrm{D}$ positions of the AMB and PLB centers were compared in the 3 directions (X, Y, and $\mathrm{Z}$ ). Also the direct 3D distance between the anatomical and computer determined centers was calculated.

\section{Results}

\subsection{Computer Assisted Placement}

The system consists of an Optotrak system, a computer and computer-tracked devices. The Optotrak system consists of 3 camera's that track the 3D positions of LED's. These are located in the non-rigid Dynamic Reference Bases (DRB's) who will be fixed to the patients femur and tibia. This will allow real-time tracking of the bones and devices and interactive digitization. 
The interface was programmed in a wizard-like fashion guiding the surgeon through the steps of acquiring the required landmarks for the template alignment. After starting the ACL-module, the first step is controlling tracking and visibility of the LED's on the DRB's on the monitor. To inform the system about the orientation of the femur and tibia, the long axis of the femur (trochantor major femur - mid condyles) and tibia (intercondylar tibia - mid ankle) have to be digitized, as is the medial-lateral axis (between the femoral epicondyles). The next step is the template alignment.

First the notch surface will be interactively digitized as a cloud of points using a computer-tracked palpation hook (Stillehook) (Fig.5.a). An algorithm uses this cloud of points to generate a 3D surface for better visualization (Fig.5.b). The same palpation hook is then used to interactively digitize the cartilage border (fig. 5.c). A cylinder fit into the notch surface through a Levenberg-Marquardt algorithm, determines template orientation. At the same time this results in the sizing of the template to the patient's knee. The apex of the cartilage border curve, as described in a cylindrical coordinate system, will find template rotation about the cylindrical axis. Since surgical orientation of the reference base on the femur is arbitrary, the system gives several different start conditions for the Levenberg-Marquardt algorithm and chooses the solution having the smallest residual. The template is then visualized as a $3 \mathrm{D}$ cylinder for the user to verify proper algorithm convergence and suggested tunnel locations (in ACL-, AMB- or PLB-center) are displayed as small 3D spheres (Fig. 5.d). The computer interface is then used to guide a computer-tracked awl to mark the drill hole point(s) in the patient's knee with a physical indent (Fig. 5.e+5.f) A K-wire is drilled into the marked point, after which the tunnel will be drilled. If wanted the drilling can also be done computer-assisted by using a tracked drill guide.

\subsection{Validation Results Template}

Table 1. The mean differences $( \pm \mathrm{sd})$ in $\mathrm{mm}$ reported for the anatomical AMB and PLB center locations and those found by numerical template fit with CAS, in 33 tested femora.

\begin{tabular}{ccccc}
\hline & 3D & X-direction & Y-direction & Z-direction \\
\hline AMB & $3.2 \pm 1.3$ & $1.3 \pm 1.2$ & $1.8 \pm 1.2$ & $1.0 \pm 0.8$ \\
PLB & $2.1 \pm 1.2$ & $1.4 \pm 1.1$ & $1.4 \pm 1.0$ & $0.7 \pm 0.6$ \\
\hline
\end{tabular}




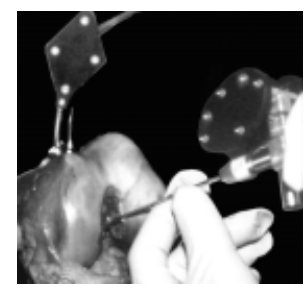

5.a.

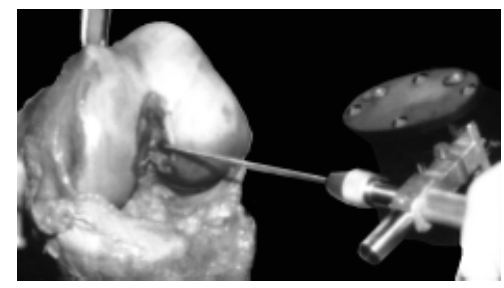

5.c.

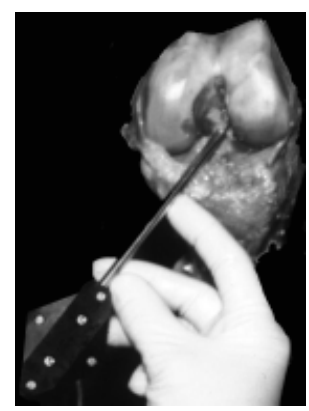

5.e.

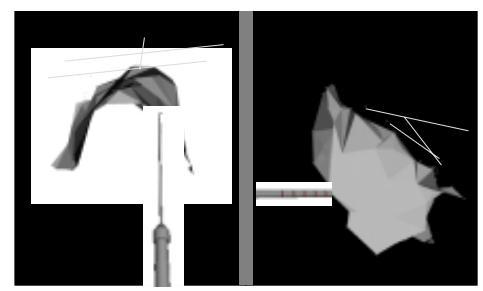

$5 . \mathrm{b}$

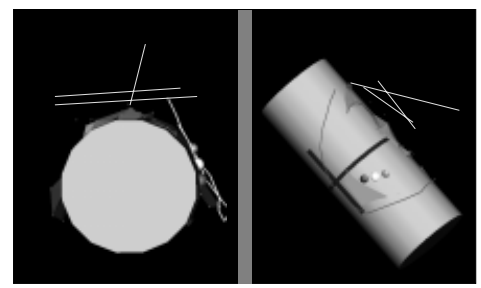

$5 . d$

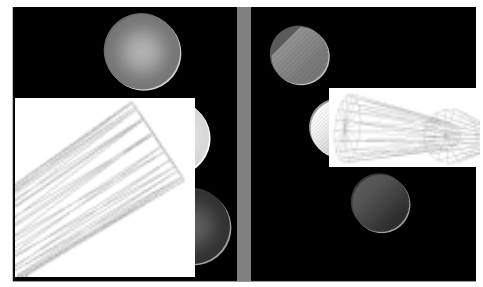

5.f.

Fig. 5. Computer-assisted placement of a double bundle ACL through $3 \mathrm{D}$ fitting of a statistically generated femoral template into individual knee geometry. A bone block with a spliced Quadriceps tendon will be fixated in a tunnel located in the anatomic ACL-center. Both slips are positioned in the correct place with the use of CAS

5.a. Picture of a tunnel view into the femoral notch: Digitizing the notch surface with the computer-tracked Stillehook. The femur is tracked through the dynamic reference base (DRB) 5.b. Notch-view (left) and lateral-medial view of the notch (right) on the monitor-screen: The digitized notch surface is $3 \mathrm{D}$ visualized for better orientation. CAS calculates the cylinderalgorithm to fit the notch surface

5.c. Picture of a tunnel view into the femoral notch: Digitizing of the cartilage border at the medial notch wall of the lateral femoral condyle with the tip of the Stillehook

5.d. Notch-view (left) and lateral-medial view of the notch (right) on the monitor-screen: CAS positions the scaled template with the anatomic gray AMB-, black PLB- and white ACL-center 5.e. Picture of a tunnel view into the femoral notch: Marking of the drill hole location in the notch with a physical indent of the computer-tracked awl

5.f. Zoomed notch-view (left) and lateral-medial view of the notch (right) on the monitorscreen: The white anatomic ACL-center is the target point for the mark made by the awl, which is visualized on the CAS monitor 


\section{Discussion}

Practical use. The wizard-like fashion of the interface is a simple and practical way of determine the insertion centers of the ACL, AMB and PLB. Algorithm convergence did not always give the right solution on the first trail on each knee. In some knees the first digitization was enough, in some it needed to be redone several times. Refining of the notch surface with additional points until the cylinder fit was visually correct, was possible for all knees. Even with several start conditions, total calculation time of the optimization algorithm was about 10-15 seconds on a Sun (Ultra 1 creator). The total extra operating time was about 10 minutes.

Application. The system was used in an in-vitro experiment with double bundle ACL-reconstruction for computer assisted anatomic femoral graft placement [12]. In the tested femora $(n=11)$, no cartilage damage or dorsal outbreak of the drilled tunnel appeared.

Computer assisted localization and placement. The additional contribution of the developed method is besides the 3D approach, the use of the CAS system in defining the location of the femoral drill hole, based on the average knee anatomy. No extra images are needed, neither the use of fluoroscopy. CAS is therefore not only used for accurate placement, but also to reproduce the location within 1-3 $\mathrm{mm}$ of the anatomical insertion center of an individual patient. It is a simple method, which enables the surgeon to place the graft where he wants it (anatomic AMB, PLB or ACL center) and to use an outside-in drill technique.

Disadvantages. The disadvantages of the system are, besides the costs and (little) extra operating time (10'), the use of the DRB's, which causes extra wounds. In chronic cases with notch deformation, the method is not fit.

Accuracy. The developed method to determine the localization of the anatomical AMB and PLB center has a high accuracy $(<2 \mathrm{~mm})$ in the separate directions. From $3 \mathrm{D}$ viewpoint the centers can be placed within 1-3 mm of its anatomical center.

Future. Further biomechanical studies are needed to evaluate the difference between "isometric" and anatomic placement.

\section{Conclusion}

The computer-program with the template is a simple and accurate guide in computerassisted 3D localization of the femoral drill hole and anatomical placement of a double bundle ACL-graft into individual knee geometry.

Acknowledgment. This study is supported by the AO Foundation, Switzerland. 


\section{References}

1. Harner C.D., Hyun Baek, G., Vogrin, T.M., Carlin, G.J., Kashiwaguchi, S., Woo, S. L-Y.: Quantitative Analysis of Human Cruciate Ligament Insertions. J Arth Rel Surg Vol. 17 (7) (1999) 741-49

2. Girgis, F.G., Marshall, J.L., Al Monajem, A.R.S.: The cruciate ligaments of the knee joint, anatomical, functional and experimental analysis. Clin Orth Rel Res Vol. 106 (1975) 216-231

3. Amis, A.A., Jakob, R.P.: Knee Surg, Sports Traumatol, Arthrosc Vol. 6 suppl 1 (1998) S2-S12

4. Bernard, M., Hertel, P., Hornung, H., Cierpinski ,Th.: Femoral insertion of the ACL, radiographic quadrant method. Am J Knee Surg Vol. 10 (1) (1997) 14-22

5. Harner, C.D., Marks, P.H., Fu, F.H., Irrgang, J.J., Silby, M.B., Mengato, R.: Anterior cruciate ligament reconstruction: endoscopic versus two- incision technique. J Arth Rel Surg Vol. 10 (1994) 502-512

6. Lintner, D..M., Dewitt, S.E., Moseley, J.B.: Radiographic evaluation of native anterior cruciate ligament attachments and graft placement for reconstruction, A cadavaric study. Am J Sports Med Vol. 24 (1) (1996) 72-78

7. Sati, M., Stäubli, H.-U., Bourquin, Y., Kunz, M., Käsermann, S., Nolte, L.-P.: Clinical Integration of New Computer Assisted Technology for Arthroscopic ACL Replacement. In: T.DiGioia (Guest Ed.), F. Fu (Ed.): Operative Techniques in Orthopaedics, Vol. 10 (1) (January 2000) 40-49

8. Stäubli, H.-U., Käsermann, S., Kunz, M., Sati, M.: Inter-operator variance of ligament placement: Endoscopic versus. Abstr 4th Intern Symp on CAOS (1999) 17

9. Klos, T.V.S., Habets, R.J.E., Banks, A.Z., Banks, S.A., Devilee, R.J.J., Cook F.F.: Computer Assistance in Arthroscopic Anterior Cruciate Ligament Reconstruction. Clin Orth Rel Res Vol. 354 (1998) 65-69

10. Picard, F., Moody, J., Jaramaz, B., GiGioia, A.M.: Computer-assisted ACL reconstruction surgery: "High tech" does not necessarily mean "high complexity". Abstr 5th Intern Symp on CAOS (2000) 22

11. Petermann, J., Schierl, M., Heeckt, P.F., Gotzen L.: The CASPAR-system (Computer Assisted Surgery Planning And Robotics) in the reconstruction of the ACL. First followup results. Abstr 5th Intern Symp on CAOS (2000) 23

12. Wymenga, A.B., Luites, J.W.H., Sati, M., Bourquin, Y., Blankevoort, L., van der Venne, R., L., Kooloos, J.G.M., Stäubli, H.-U.: Computer-Assisted Anatomical Placement of a Double-Bundle ACL through 3D-Fitting of a Statistically Generated Femoral Template Into Individual Knee Geometry. Abstr 5th Intern Symp on CAOS (2000) 24 